\title{
Regulation of PD-L1 Expression by Histone Deacetylase Inhibitor SAHA in Lung Cancer Cells
}

\author{
Umamaheswari Natarajan ${ }^{1}$, Thiagarajan Venkatesan ${ }^{1}$, Jayanta Kumar Das ${ }^{1}$ and Appu Rathinavelu ${ }^{1,2, *}$ \\ ${ }^{1}$ Rumbaugh-Goodwin Institute for Cancer Research, Nova Southeastern University, Fort Lauderdale, FL - 33314, USA \\ ${ }^{2}$ College of Pharmacy, Health Professions Division, Nova Southeastern University, Fort Lauderdale, FL - 33314, USA
}

*Corresponding author: Appu Rathinavelu, Rumbaugh-Goodwin Institute for Cancer Research, Nova Southeastern University, USA

\section{ARTICLE INFO}

Received: 画 July 07, 2019

Published: 蔧 July 17, 2019

Citation: Umamaheswari Natarajan, Thiagarajan Venkatesan, Jayanta Kumar Das, Appu Rathinavelu. Regulation of PD-L1 Expression by Histone Deacetylase Inhibitor SAHA in Lung Cancer Cells. Biomed J Sci \& Tech Res 19(4)-2019. BJSTR. MS.ID.003348.

Keywords: SAHA; PD-L1 Expression; Lung Cancers; p21; HDAC Inhibitor

\begin{abstract}
The expression of PD-L1 is checkpoint indicator indicative of immunosuppression and cause for poor prognosis in several cancers. The effects of PD-L1 are mediated through its binding to the Programmed Cell Death-1 (PD-1) receptor, which is expressed on lymphoid and non-lymphoid derived cells. The binding of PD-L1 to the PD-1 receptor mediates checkpoint inhibition to regulate peripheral T-cell responses. Therefore, determining the mechanisms of regulation of the PD-L1 expression is very important for understanding the impacts of immunosuppressive microenvironment, and is also crucial for the purpose of reactivating the T-cells that can lead to enhanced tumor attack. Therefore, we analyzed relieving the T-cell from the checkpoint inhibition. The expression of PD-L1 was analyzed in various cancer cells and the highest-level of expression was found in HCC827 (Lung Adenocarcinoma) cells followed by H460 (Large Cell Lung Cancer), H226 (Squamous Cell Carcinoma), H1975 (Non-Small Cell Lung Cancer) and H1568 (Non-Small Cell Lung Cancer). Relatively lower level expressions were found in H23 (Non-Small Cell Lung Cancer) and H522 (Non-Small Cell Lung Cancer) cell lines. To determine the effects of HDAC (Histone Deacetylase) inhibitor SAHA (suberoylanilide hydroxamic acid), we analyzed the expression levels of PD-L1 in HCC827 cells. The treatment of SAHA $(0.5-10 \mu \mathrm{M})$ was able to reduce the expression of PD-L1 in dose dependent manner within $24 \mathrm{hrs}$. In addition to decreasing the expression of PD-L1, SAHA treatment was sable to produce decreases in EGFR and pEGFR levels also in HCC827 cells following SAHA treatment. Treatment of HCC827 cells with SAHA increased the levels of acetyl-H2B, and acetyl-H3. Since acetylation mediated unwinding of the DNA is typically responsible for increased expression of p21/CDKN1A gene, SAHA treatment elevated the levels of p21 WAF1/CIP1 also. It is suspected that the decrease in the PD-L1 levels may be due to reduced transcription of the CD274 gene that is coding for PD-L1. However, it is not clear whether the decrease is because of p21 ${ }^{\mathrm{WAF} 1 / \mathrm{CIP} 1}$ mediated negative control on the transcription or due to some other mechanism. In addition to regulating PD-L1 expression in HCC827 cells that were growing in monolayer, SAHA treatment for 7 days induced significant reduction in the volume of $\mathrm{H} 1975$ cells that were grown as spheroids. Additional studies are required to fully understand the actual mechanisms that may be involved in the regulation of PD-L1 expression in lung cancer cells.
\end{abstract}

\section{Introduction}

Various pathways can be utilized by cancers to avoid recognition by the immune system to escape from the T-cell attack in the body. Actively involved in this process are the pairs of inhibitory receptors and ligands that are expressed on T-cells, Antigen Presenting Cells (APCs) and other types of immune cells, which are collectively termed as 'immune check points'. In order to escape from the detrimental effects of the immune responses that occur in response to $\mathrm{T}$ cell activation, the checkpoint signaling is often inhibited by the cancer cells, through binding of PD-1 receptor by specific cell surface ligands such as PD-L1 that are expressed on cancer cells [1]. The PD-1 receptor is mainly expressed on antigen-sensitized memory T-cells in peripheral tissues [2]. It can also be expressed on B-cells, monocytes, Dendritic Cells (DCs) and Natural Killer (NK) cells. The levels of PD-1 is rapidly up-regulated after exposure to cognate antigens but is down-regulated upon antigen clearance. Thus, the PD-1 receptors that are expressed on naïve or 
memory T-cells in lymphoid tissues, serve as attractive target for PD-L1 binding, which leads to rapid blockade of the effector function of T-cells. Thus, the PD-1 receptor and its ligands PD-L1 or PDL2, which are the members of the CD28 and B7 families, play critical roles in $\mathrm{T}$ cell co-inhibition and exhaustion. In addition, overexpression of PD-L1 on tumor cells correlates with poor treatment outcome in several human cancers $[3,4]$. Therefore, monoclonal antibodies have been developed for the checkpoint rescue therapy, to induce the antitumor immunity by activating the suppressed T-cells. Some of the anti-CTLA-4 and anti-PD-L1 antibodies have led to extraordinary increases in overall survival of patients, in various malignancies [5,6]. Consequently, greater appreciation for the utility of anti-PD-1 or anti-PD-L1 therapy has been developing in the recent times.

Though PD-L1 is commonly elevated in cancer cells, the mechanisms that control PD-L1 expression during the pathogenesis of cancer remains unclear. Some of the recent studies have identified the pro-survival signaling pathway as a common mechanism by which upregulation of PD-L1 can be accomplished in cancer cells. Nevertheless, there is a necessity to comprehensively understand the molecular and cellular interactions between PD-1 and PD-L1 and the extent by which this axis is involved in influencing the immunity towards a variety of cancer types [7-10]. To further advance the existing knowledge related to PD-L1 mediated immune-escape, we analyzed the expression levels of PD-L1 in lung cancer cells after treating them with SAHA. The results presented here are pointing towards a very valuable strategy.

\section{Materials and Methods}

\section{Reagents}

PD-L1 antibody was purchased from R\&D Systems (Minneapolis, MN, USA). Histone Deacetylase (HDAC) inhibitor SAHA was purchased from Selleckchem (Houston, TX, USA). p21 WAF1/CIP1, EGFR, pEGFR, AC.H2B, and AC.H3 antibodies (1:1000) were purchased from Cell Signaling Technology (Danvers, MA, USA).

\section{Cell Lines}

All cell lines were cultured in RPMI-1640, It were supplemented with 10\% Fetal Bovine Serum (FBS) and 1\% ABAM. The cells were cultured in a humidified atmosphere of 95\% air and 5\% CO2. Whenever needed, the cells were treated individually with $0.5,2.5$, $5.0,7.5,10.0(\mu \mathrm{M})$ SAHA

\section{Western Blot Analysis}

Western blotting was used to determine the levels of PD-L1, p21 ${ }^{\text {WAF1/CIP1 }}$, EGFR, pEGFR and Acetyl-Histone H2B H3 expressions after SAHA treatment. The resolved proteins were blotted onto nitrocellulose membrane and then the protein bands were detected using the Enhanced Chemiluminescence (ECL) system and then the images we captured using the UVP image analyzer. Densitometric analyses of the bands in the western blots were performed using the ImageJ program (NIH Image, Bethesda, MD)

\section{Three-Dimensional (3d) Spheroid Assay with H1975 Lung Cancer Cells}

For spheroid formation, one hundred lung cancer cells (H1975) per well were seeded in $200 \mu \mathrm{L}$ serum-free RPMI 1640 culture medium supplemented with B27 in an ultra-low-attachment 24 well plate (Corning Inc., Lowell, MA) for $72 \mathrm{hrs}$. The dose-dependent effect of SAHA, a Histone Deacetylase Inhibitor (HDACi) was determined by treating the spheroids with different concentrations $(0,1.25,2.5,7.5,10,20 \mu \mathrm{M})$ of SAHA. Spheroids were grown for 7 days at $37^{\circ} \mathrm{C}$ in a humidified atmosphere with $5 \% \mathrm{CO}_{2}$. A total of 15 spheroids' diameters were counted in each experimental group. Data were calculated with mean values \pm SD for comparisons among control and different treatment groups.

\section{Results and Discussion}

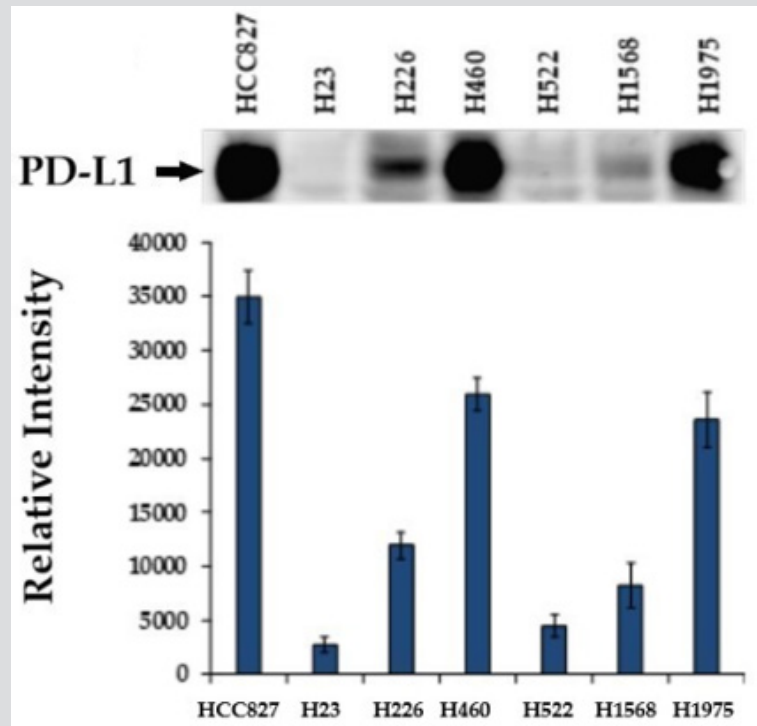

Cancer Cell Lines

Figure 1: Expression of PD-L1 expression in HCC827, H23, H226, H460, H522, H1568 and H1975 cancer cell lines.

When we analyzed the expression levels of PD-L1 our results confirmed the highest-level of expression in HCC827 lung cancer cells (Figure 1). In addition, H460, H1975 (Lung Cancer), cells were also found to express PD-L1 in high levels (Figure 1). Relatively, lower level expression of PD-L1 was detected in H23, H1568 and H522 cells. Since HCC827 cells showed the highest levels of PD-L1, the regulation of its level was assessed using these cells. The cells were treated with SAHA up to $10 \mu \mathrm{M}$ concentration and the HDAC inhibitor showed significant reduction of PD-L1 levels starting from $2.5 \mu \mathrm{M}$ concertation (Figure 2). SAHA was also able to significantly elevate the levels of p21 $1^{\text {WAF1/CIP1 }}$ also in HCC827 cells (Figure 2) without impacting the levels of p53 (data not shown). Thus, our results clearly suggested that SAHA treatment could significantly elevate $21^{\text {WAF1/CIP1 }}$ expression in HCC827 lung cancer cells, following 
SAHA treatment while the PD-L1 expression was downregulated. Concomitantly, the levels of EGFR and pEGFR were also reduced in HCC827 cells following SAHA treatment (Figure 3). When the levels of acetylated H2B was assessed in SAHA treated HCC827 cells, the acetyl-H2B level was found to be elevated significantly, as shown in (Figure 4). This result correlated inversely with the decreased levels of PD-L1 and positively with the p21 $1^{\text {WAF1/CIP1 }}$ levels. Since acetylation mediated unwinding of the DNA is typically responsible for the increased expression of genes such as p21 ${ }^{\text {WAF1/CIP1 } 1}$, during HDAC inhibition, it was occurring as per our expectations in the HCC827 lung cancer cells also. Furthermore, it is suspected that, the decrease in the PD-L1 levels observed in HCC827 cells may be due to suppression of the CD274 gene transcription following hyperacetylation of H2B. However, it is not certain whether the decrease in PD-L1 protein levels observed following HDAC inhibition is because of the direct effects of $\mathrm{p} 21^{\mathrm{WAF} 1 / \mathrm{CIP} 1}$ mediated pathway on the transcription / degradation of the PD-L1 proteins or purely due to the impact of acetyl-H2B on the transcription mechanism. In addition to decreasing the expression of PD-L1, the SAHA treatment was able to reduce the EGF and pEGFR levels also. However, it is not clear whether $\mathrm{p} 21$ is responsible for this reduction. Also, incubation of H1975 lung cancer cells growing as spheroids in 3D format, with SAHA for 7 days in the concentration range of $1.25 \mu \mathrm{M}-20.0 \mu \mathrm{M}$, yielded interesting results. SAHA treatment was able to produce significant reduction in the volume of the spheroids over a period of 7 days, with complete disruption seen in $7.5 \mu \mathrm{M}$ concentration to $20 \mu \mathrm{M}$ concentrations (Figure 5). It is not clear whether there is any link between the cytoreductive effect and the PD-L1 expression in H1975 cells. Additional studies, such as use of p21 WAF1/CIP1 inhibitors or EGFR inhibitors are necessary to fully understand the interplay between $21^{\mathrm{WAF} 1 / \mathrm{CIP} 1}$, pEGFR and PD-L1 and their impact on the volume of cells growing as spheroids.

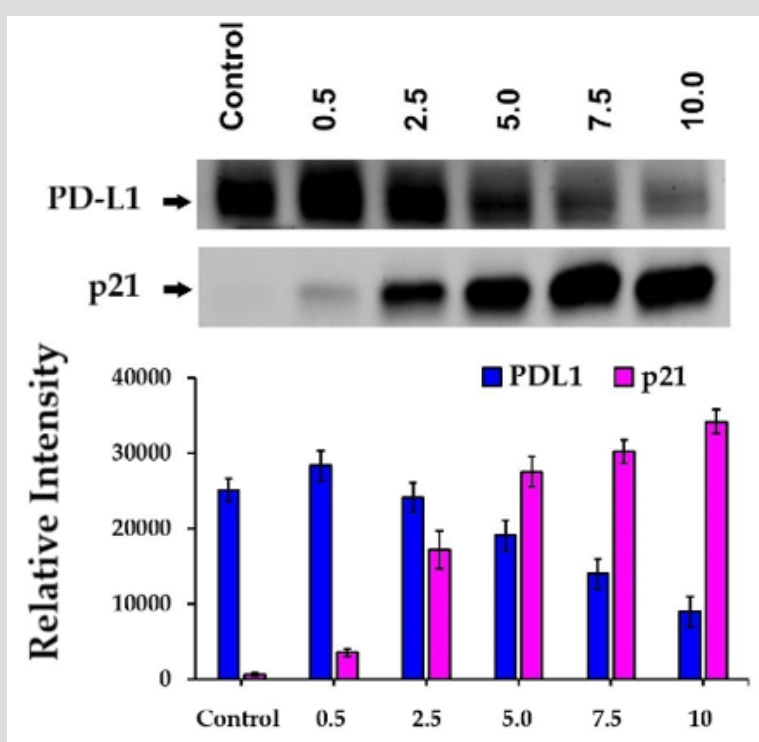

Concentration of SAHA $(\mu \mathrm{M})$

Figure 2: Effect of different concentrations of SAHA (0.5 $10 \mu \mathrm{M})$ on PD-L1 and p21 ${ }^{\mathrm{WAF} 1 / \mathrm{CIP} 1}$ expressions in HCC 827 lung cancer cells.

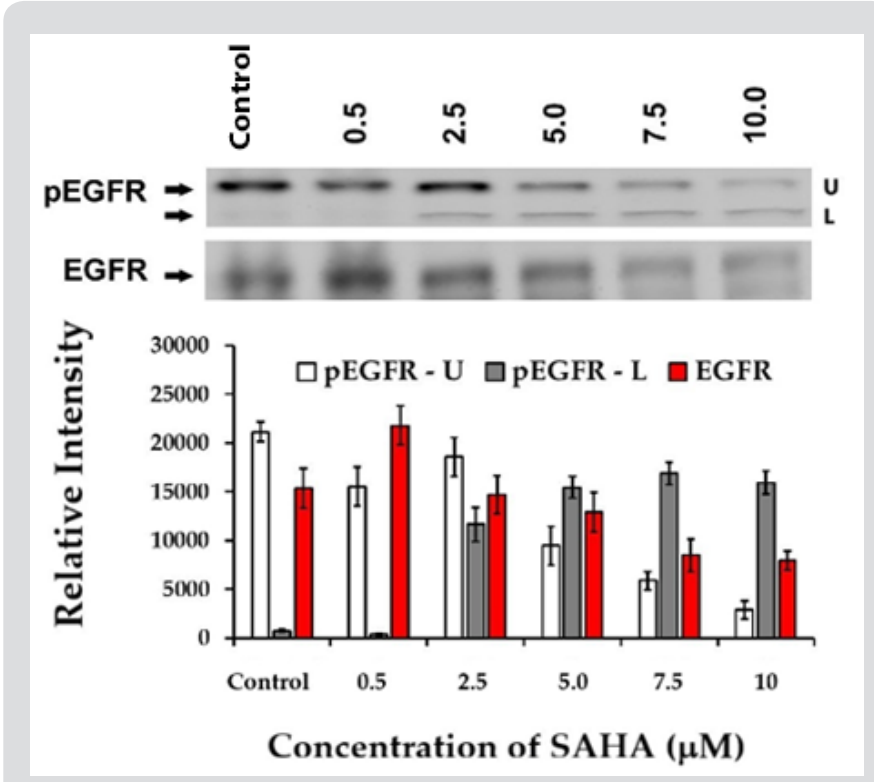

Figure 3: Effect of different concentrations of SAHA (0.5$10 \mu \mathrm{M})$ on pEGFR and EGFR expressions in HCC827 lung cancer cells.

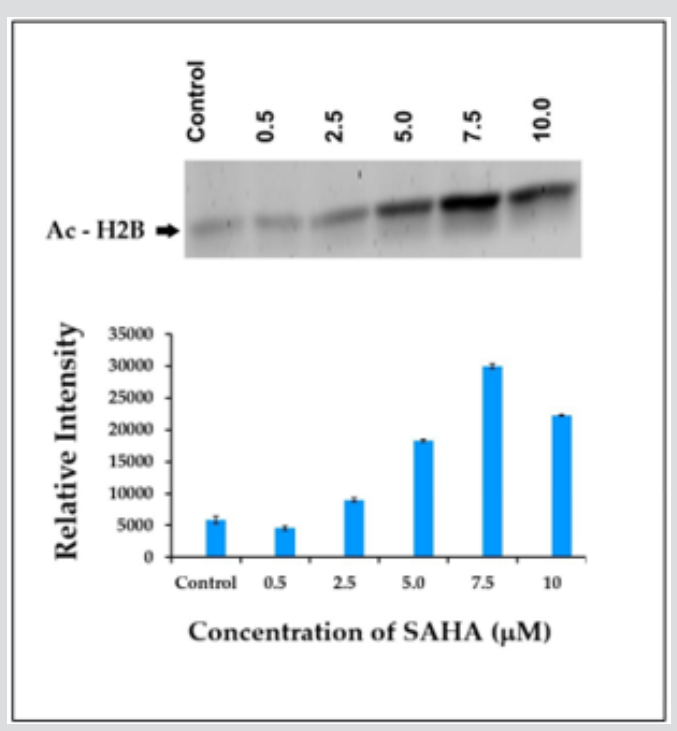

Figure 4: Effect of different concentrations of SAHA (0.5$10 \mu \mathrm{M})$ on the acetylation status of H2B in HCC827 cells.

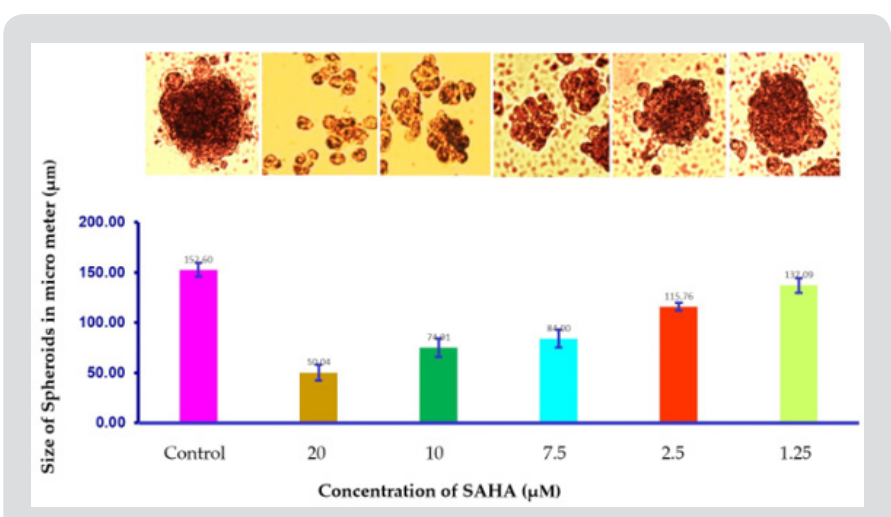

Figure 5: Effect of different concentrations of SAHA (1.25 - $20 \mu \mathrm{M}$ ) after 7 days on the growth volume of H1975 cells that were growing as spheroids. 
So far, our preliminary results indicate a very important consequence of HDAC inhibition in relation to the PD-L1 levels. Very recently, the regulation of PD-L1 by HAT1 (Histone Acetyltransferase 1) was reported to occur in Pancreatic Ductal Adenocarcinoma Cells (PDAC). In addition to promoting the cell proliferation that was leading to poor prognosis, the elevation of HAT1 level was reported to increase PD-L1 overexpression also [11]. However, our results suggest that, in HCC827 lung cancer cells, hyperacetylation of H2B following HDAC inhibition might lead to inhibition of PD-L1 expression. Since hyperacetylation of histones is reported to produce both down regulation and upregulation of genes, it is important to verify whether the decrease in the PD-L1 expression observed in HCC827 cells is really a direct consequence of hyperacetylation mediated suppression of gene transcription or due to some other mechanism.

\section{Acknowledgement}

This research was supported by the Royal Dames of Cancer Research Inc., Fort Lauderdale, Florida.

\section{References}

1. Korman AJ, Peggs KS, Allison JP (2006) Checkpoint blockade in cancer immunotherapy. Adv Immunol 90: 297-339.

2. Pauken KE, Wherry EJ (2015) Overcoming T cell exhaustion in infection and cancer. Trends Immunol 36(4): 265-276.

3. Parry RV, Chemnitz JM, Frauwirth KA, Lanfranco AR, Braunstein I, et al. (2005) CTLA-4 and PD-1 receptors inhibit T-cell activation by distinct mechanisms. Mol Cell Biol 25(21): 9543-9553.

\section{ISSN: 2574-1241}

DOI: 10.26717/BJSTR.2019.19.003348

Appu Rathinavelu. Biomed J Sci \& Tech Res

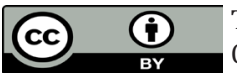

This work is licensed under Creative Commons Attribution 4.0 License

Submission Link: https://biomedres.us/submit-manuscript.php
4. Ohaegbulam KC, Assal A, Lazar-Molnar E, Yao Y, Zang X (2015) Human cancer immunotherapy with antibodies to the PD-1 and PD-L1 pathway. Trends Mol Med 21(1): 24-33.

5. Drew MP (2012) The blockade of immune checkpoints in cancer immunotherapy. Nat Rev Can 12(4): 252-264.

6. Pico CY, Choudhury A, Kiessling R (2015) Checkpoint blockade for cancer therapy: revitalizing a suppressed immune system. Trends Mol Med 21(8): 482-491.

7. Joseph Grosso, David Inzunza, Qiuyan Wu, Jason Simon, Parul Singh, et al. (2013) Programmed death-ligand 1 (PD-L1) expression in various tumor types. J Immunother Cancer 1(Suppl 1): P53.

8. Iwai Y, Ishida M, Tanaka Y, Okazaki T, Honjo T, et al. (2002) Involvement of PD-L1 on tumor cells in the escape from host immune system and tumor immunotherapy by PD-L1 blockade. Proc Natl Acad Sci 99(19): 12293-12297.

9. Liu J, Hamrouni A, Wolowiec D, Coiteux V, Kuliczkowski K, et al. (2007) Plasma cells from multiple myeloma patients express B7-H1 (PD-L1) and increase expression after stimulation with IFN- and TLR ligands via a MyD88-, TRAF6-, and MEK-dependent pathway. Blood 110(1): 296304.

10. Thompson RH, Gillett MD, Cheville JC, Lohse CM, Dong H, et al. (2004) Costimulatory B7-H1 in renal cell carcinoma patients: Indicator of tumor aggressiveness and potential therapeutic target. Proc Natl Acad Sci 101(49): 17174-17179.

11. Fan P, Zhao J, Meng Z, Wu H, Wang B, et al. (2019) Overexpressed histone acetyltransferase 1 regulates cancer immunity by increasing programmed death-ligand 1 expression in pancreatic cancer. J Exp Clin Cancer Res 38: 47.

$\begin{array}{ll}\text { BIOMEDICAL } & \text { Assets of Publishing with us } \\ \text { RESEARCHES } & \text { - Global archiving of articles } \\ & \text { - Immediate, unrestricted online access } \\ & \text { - Rigorous Peer Review Process } \\ \end{array}$

